\title{
Standard Diffusion-Weighted Imaging in the Brain Can Detect Cervical Internal Carotid Artery Dissections
}

(D) G. Adam, (D). Darcourt, (D) M. Roques, (D). Ferrier, (D). Gramada, (D). Meluchova, (D). Patsoura, (D) A. Viguier, (D). Cognard, (D) V. Larrue, and (DF. Bonneville

\begin{abstract}
BACKGROUND AND PURPOSE: The ICA is the most common site of cervical artery dissection. Prompt and reliable identification of the mural hematoma is warranted when a dissection is clinically suspected. The purpose of this study was to assess to capacity of a standard DWI sequence acquired routinely on the brain to detect dissecting hematoma related to cervical ICA dissections.
\end{abstract}

MATERIALS AND METHODS: This was a retrospective study of a cohort of 110 patients younger than 55 years of age (40 women; mean age, 46.79 years) admitted at the acute phase of a neurologic deficit, headache, or neck pain and investigated by at least a standard 3T diffusion-weighted sequence of the brain. Among them were 50 patients (14 women; mean age, 46.72 years) with subsequently confirmed ICA dissection. In the whole anonymized cohort, both a senior and junior radiologist separately assessed, on the DWI sequences only, the presence of a crescent-shaped or circular hypersignal projecting on the subpetrosal segment of the ICA arteries, assuming that it would correspond to a mural hematoma related to an ICA dissection.

RESULTS: The senior radiologist found 46 subpetrosal hyperintensities in $43 / 50$ patients with ICA dissection and none in patients without dissection (sensitivity, $86 \%$; specificity, 100\%). The junior radiologist found 48 subpetrosal hyperintensities in $45 / 50$ patients with dissection and none in patients without dissection (sensitivity, $90 \%$; specificity, $100 \%$ ).

CONCLUSIONS: In our cohort, a standard DWI sequence performed on the brain at the acute phase of a stroke or for a clinical suspicion of dissection detected nearly $90 \%$ of cervical ICA dissections.

ABBREVIATION: FS = fat-saturated

ervical artery dissection is defined by the spontaneous or posttraumatic onset of a hematoma in the wall of an artery. The cervical ICA is the most common site for dissection of supra-aortic trunks. ${ }^{1}$ While cervical artery dissections are responsible for nearly $25 \%$ of ischemic strokes in young or middle-aged adults, ${ }^{2}$ dissections may be diagnosed without or before the onset of a stroke. These ischemic complications occur in $82 \%$ of patients in the first week following dissection. ${ }^{3}$ It is, therefore, essential to be able to diagnose cervical artery dissection as soon as possible following the appearance of a mural hematoma to implement an adapted treatment, medical or endovascular, and

Received April 4, 2019; accepted after revision November 27

From the Departments of Diagnostic and Therapeutic Neuroradiology (G.A., J.D. M.R., M.F., R.G., Z.M., S.P., C.C., F.B.) and Neurology (A.V., V.L.), Centre Hospitalier Universitaire Toulouse, Pierre-Paul Riquet Hospital, Toulouse, France.

Please address correspondence to Fabrice Bonneville, MD, CHU Toulouse, PierrePaul Riquet Hospital, Place du Docteur Baylac, TSA 4003131059 Toulouse, France; e-mail: bonneville.f@chu-toulouse.fr

Indicates article with supplemental on-line table.

http://dx.doi.org/10.3174/ajnr.A6383 reduce the risk of thromboembolic or hemodynamic complications. ${ }^{4}$ In this context of dissection and in case of stroke due to a large-vessel occlusion, noninvasive imaging of the extra- and intracranial arteries is essential for planning, if necessary, an endovascular recanalization strategy, both cervical and cerebral, ${ }^{5,6}$ because tandem occlusions may occur and can be treated at the same time. ${ }^{7}$

MR imaging allows the diagnosis of cervical artery dissection by directly visualizing the wall hematoma through fat-saturated (FS) T1WI. It appears as a crescent-shaped hypersignal around an eccentric residual arterial lumen. This T1 hyperintensity is associated with the presence of intracellular and extracellular methemoglobin in the hematoma. ${ }^{8}$ Because these hemoglobindegradation products only appear 72 hours after the formation of the hematoma, T1 hypersignal is only visible after this period. Before 72 hours, oxyhemoglobin and deoxyhemoglobin appear isointense on T1-weighted sequences; this feature makes diagnosis difficult in the acute phase and can be the cause of false-negatives. ${ }^{9}$ The sensitivity of the FS T1WI is, therefore, more reliable after the first 72 hours of the onset of a dissection. ${ }^{10}$ 

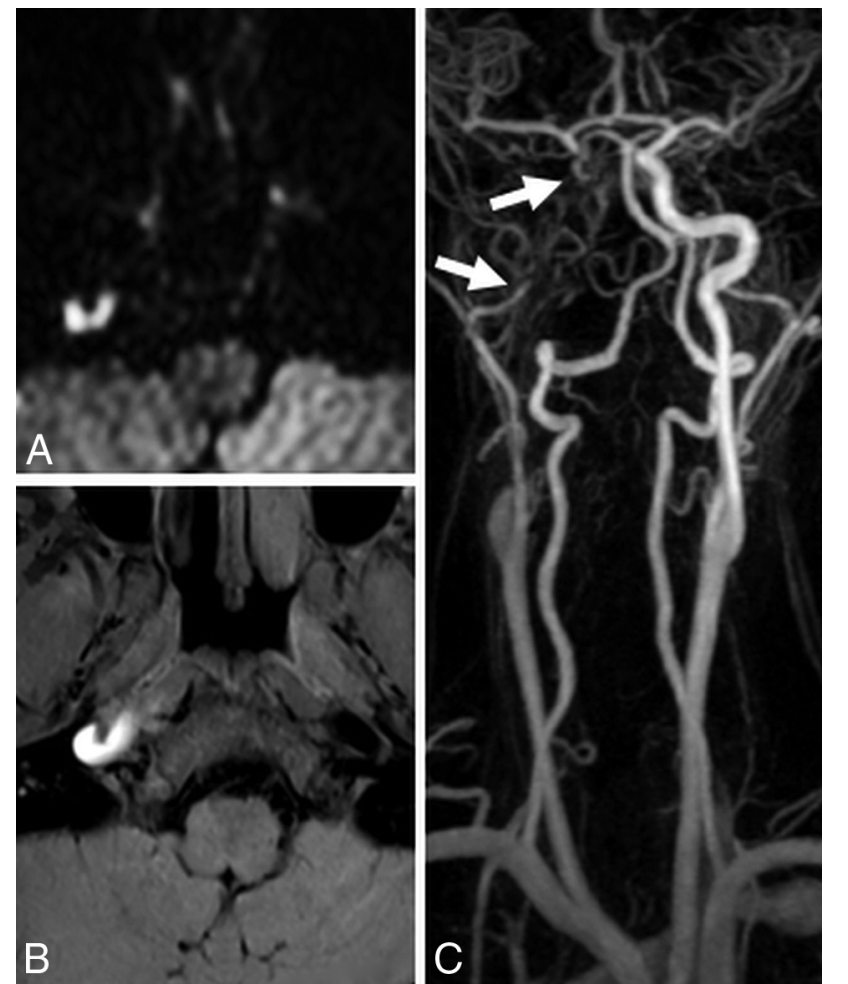

FIG 1. DWI $(A)$ and FS TIWI (B) show a crescent-shape hypersignal of a mural hematoma of a right carotid artery dissection. MR angiography $(C)$ shows the subsequent near-occlusion stenosis of the arterial lumen.

DWI is generally the first sequence acquired during a brain MR imaging performed to explore an acute neurologic deficit to identify early signs of cerebral ischemia. On this sequence, acute cerebral hemorrhage also appears hyperintense $\mathrm{e}^{11}$ and wall hematomas of cervical artery dissections are sometimes also visible as a hypersignal. ${ }^{12,13}$ Brain MR imaging usually covers the subpetrous segment of the ICA, where the wall hematoma of the dissections is most frequently seen. ${ }^{1}$

In our experience, DWI shows a crescent or circular hypersignal in the projection of the subpetrous ICA in cases of dissection (Fig 1), even during the hyperacute phase of a stroke, while FS T1WI is usually not acquired, to minimize the overall acquisition time of the examination, or the findings were negative because it was performed too early.

The aim of this work was to evaluate the capacity of DWI alone, without knowledge of other sequences, to detect wall hematomas of ICA dissections.

\section{MATERIALS AND METHODS \\ Patients}

From June 2014 to December 2016, we retrospectively included 50 consecutive patients younger than 55 years of age from a register of our university hospital (14 women; mean age, 46.72 years; range, 21-65 years) with a final diagnosis of carotid dissection. This diagnosis was confirmed secondarily, either by Doppler sonography when an intimal flap or a parietal hematoma was visible (10 patients, $20 \%$ ), or by MR imaging showing a hyperintense parietal wall hematoma on FS T1WI (22 patients, 44\%), or finally during follow-up showing either restoration to the original condition of the caliber of the cervical ICA, which presented at the acute phase with a postbulbar irregular stenosis (18 patients, $36 \%$ ), or the development of a pseudoaneurysm (14 patients, $28 \%)$. Patients were admitted to the neurovascular emergency department due to neck pain (18 patients, 36\%), headache (35 patients, $70 \%$ ), Horner syndrome (28 patients, $56 \%$ ), or neurologic deficit (30 patients, 60\%) such as visual disturbances (14 patients, 28\%), speech disorders (12 patients, 24\%), or sensory (13 patients, $26 \%$ ) or motor disorders (24 patients, $48 \%$ ) (On-line Table). The average NIHSS score on arrival at the neurovascular emergency department was 5 (range, 0-24). The patients were examined with at least routine DWI on a $3 \mathrm{~T}$ MR imaging. Among these 50 patients, 28 patients (56\%) had acute cerebral ischemic injury on initial MR imaging. The time between the onset of any symptoms reported by the patient (ie, neck pain, headaches, or neurologic deficit), considering that it might correspond to the onset of the internal carotid artery wall hematoma, and acquisition of MR imaging was calculated for each patient.

A control group of 60 individuals had the same distribution in sex and age. This group consisted of 2 subgroups: 35 patients (11 women; mean age, 47.91 years; range, 20-65 years) who had an ischemic stroke unrelated to carotid dissection and 25 patients (15 women; mean age, 45.36 years; range, $21-65$ years) who had a clinical suspicion of carotid dissection due to aggressive symptoms such as neck pain or Horner syndrome, but for whom the dissection diagnosis was rejected by the complete radiologic assessment. The final diagnoses for those patients were ischemic stroke (35/60 patients, $58 \%$ ); functional disorder (9/60 patients, $15 \%)$; transient ischemic attack (7/60 patients, $12 \%)$; migraine with aura (5/60 patients, $8 \%$ ); ear, nose, and throat pathology (2/60 patients, $3 \%)$; rupture of an aneurysm (1/60 patients, $2 \%)$; and a vasovagal episode (1/60 patients, $2 \%)$. The control population was admitted during the same period and underwent the same 3T cerebral DWI as the 50 patients with carotid dissection not have been consecutively enrolled to match with the dissection group in age and sex and presence of stroke on DWI.

The 50 patients with a final diagnosis of carotid dissection and the 60 controls without dissection showed no significant difference regarding the presence of ischemic stroke: Twenty-eight of $50(56 \%)$ patients with carotid dissection had an ischemic stroke, while $35 / 60$ (58\%) patients from the control group had one $(P=.4)$. Two patients $(4 \%)$ had a transient ischemic attack in the "dissection" group. In the control group, 35 patients (58\%) had acute cerebral ischemic lesions and 7 patient (12\%) had a transient ischemic attack.

\section{MR Imaging}

All brain MRIs included at least 1 DWI sequence with 4-mmthick sections on 3T magnets, either a Magneton Skyra (Siemens, Erlangen, Germany) (TR/TE: 5300/96 ms; matrix: $110 \times 110$; acquisition time: 53 seconds) or an Achieva TX (Philips Healthcare, Best, the Netherlands) (TR/TE: 2756/57 ms; matrix: $112 \times 89$; acquisition time: 33 seconds), from the vertex to the upper part of the second cervical vertebra, including the distal, subpetrous cervical segment of the cervical internal carotid arteries.

AJNR Am J Neuroradiol 41:318-22 Feb 2020 www.ajnr.org 

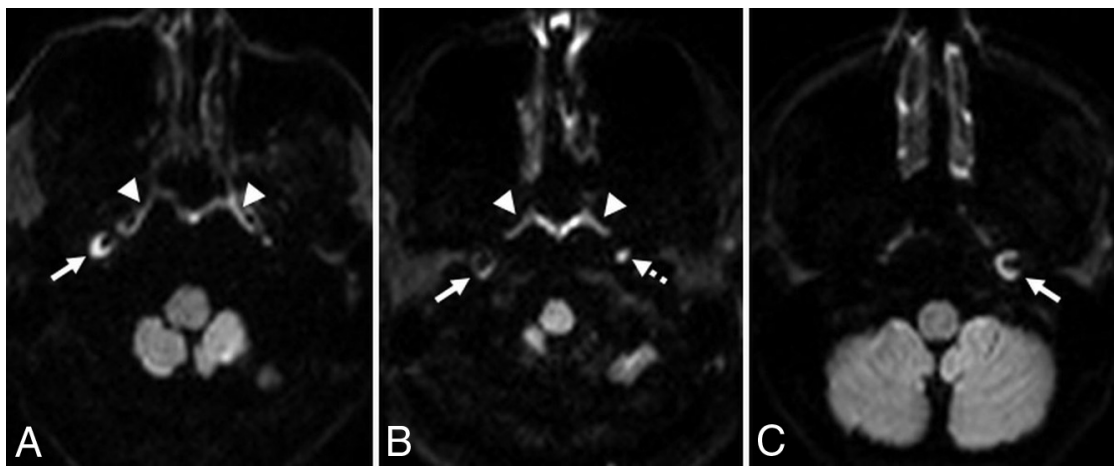

FIG 2. DWI in 3 different patients with cervical internal carotid artery dissections. Crescentshaped hypersignal of wall hematomas ( $A-C$, solid arrows), ring-shaped hypersignal of a wall hematoma ( $B$, solid arrow), linear hyperintensity of pharyngeal lymphoid tissue ( $A$ and $B$, arrowheads), and nodular hypersignal of a ganglion ( $B$, dashed arrow).

\section{MR Imaging Analysis}

The DWIs of patients and controls were isolated from the rest of the examinations, grouped together, anonymized, randomized, and randomly numbered.

Two observers, a senior radiologist (F.B.) and a junior radiologist (G.A.), with no knowledge of the patients or controls, the other sequences, and the clinical information, independently analyzed the DWI sequences separately. They had to determine whether a hypersignal with a crescent or ring shape suggestive of a wall hematoma was present in the projection of the distal cervical internal carotid arteries (Fig 2).

Other DWI high signal intensities, either located at a distance from the subpetrous segments of internal carotid arteries or of different shapes, were not retained. In particular, pharyngeal lymphoid tissue and lymph nodes, respectively, in the form of linear and nodular hypersignals, were not taken into account (Fig 2).

In a second step, among patients with dissections, after anonymity was waived, we calculated the time between the onset of symptoms and MR imaging; and when FS T1WI was performed, we compared it visually with the DWI sequence to evaluate their respective capacities in detecting a wall hematoma. Among the 50 patients with dissections, we also analyzed the shape of the DWI hyperintensities of those who had an occlusion based on MRA or CTA data (On-line Table 1).

\section{Statistical Analysis}

We calculated the sensitivity and specificity of the crescent- or ring-shaped DWI hypersignal along the expected wall of the subpetrosal ICA for the detection of carotid dissections. A $\kappa$ coefficient was calculated to measure the interobserver agreement between the senior and the junior radiologists in the detection of crescent- or ring-shaped DWI hyperintensites.

\section{RESULTS}

The senior radiologist found 46 abnormal DWI hyperintensities in the projection of the distal portion of cervical internal carotid arteries in 43/50 patients with carotid artery dissection (bilateral hypersignal for 3 patients) and none in patients without dissection (sensitivity, 86\%, specificity 100\%). The junior radiologist found 48 abnormal DWI hyperintensities in the distal portion of internal carotid arteries in 45/50 patients with carotid dissection (bilateral hypersignal for 3 patients) and none in patients without dissection (sensitivity $90 \%$; specificity $100 \%$ ).

The $\kappa$ coefficient was calculated at 0.99 , meaning excellent interobserver agreement.

Finally, after consensus, 90\% (45/ 50) of patients with dissections could be correctly diagnosed due to the DWI sequence alone, and none of the subjects of the control group were misclassified. For patients who had a dissection, the time between the onset of their symptoms and their MR imaging was $<24$ hours for $24 / 50$ patients (48\%), between 24 and 72 hours for $11 / 50$ patients (22\%) and $>72$ hours for $15 / 50$ patients (30\%).

FS T1WI was part of the initial MR imaging protocol in $26 / 50$ (52\%) patients with dissection. For these MR images, the wall hematoma appeared hyperintense on both DWI and FS T1WI in $20 / 26$ (77\%) patients. It was visible only on DWI in $4 / 26$ (15\%) patients (Fig 3). These MR images were obtained, on average, 15 hours 20 minutes after the onset of symptoms (range, 80 minutes to 32 hours). On the other hand, the hyperintensity of the wall hematoma was only visible on FS T1WI in 2/26 (8\%) patients; these MRIs were performed, on average, 6 days after the onset of symptoms (5-7 days).

\section{DISCUSSION}

The primary result of our study is that $90 \%$ of cervical ICA dissections could be detected by a standard, routine DWI sequence, acquired of the brain in the event of clinical suspicion of ischemic stroke or cervical artery dissection. Other authors ${ }^{1}$ had already investigated the possibility of diagnosing a dissection of the upper cervical portions of the internal and vertebral carotid arteries by analyzing 5 different brain sequences (sagittal T1 WI, axial FLAIR imaging, axial gradient recalled-echo T2-weighted imaging, DWI, and 3D time-of-flight angiography of the circle of Willis). In that study, the dissection diagnosis was made if the hematoma was seen on 3 different sequences. The authors showed that 59/77 (77.6\%) patients with a cervical artery dissection included in the field covered during the brain acquisition had a correct diagnosis using this method. The diagnosis was more frequent for carotid dissections (54/58 patients, 93.1\%) than for vertebral dissections (5/19 patients, $26.3 \%$ ). The sensitivity of each sequence assessed independently for detecting wall hematomas was not indicated. Our study shows comparable sensitivity of the DWI sequence alone in the detection of ICA dissections, independent of FS T1WI in particular.

However, contrary to this previous study, we analyzed not only the carotid dissections that were included in the field of acquisition of the DWI, but we included all carotid dissections without prejudging their locations and their extensions to subpetrous segments of the cervical ICA. Our results would, therefore, suggest that most carotid dissections reach the distal, 

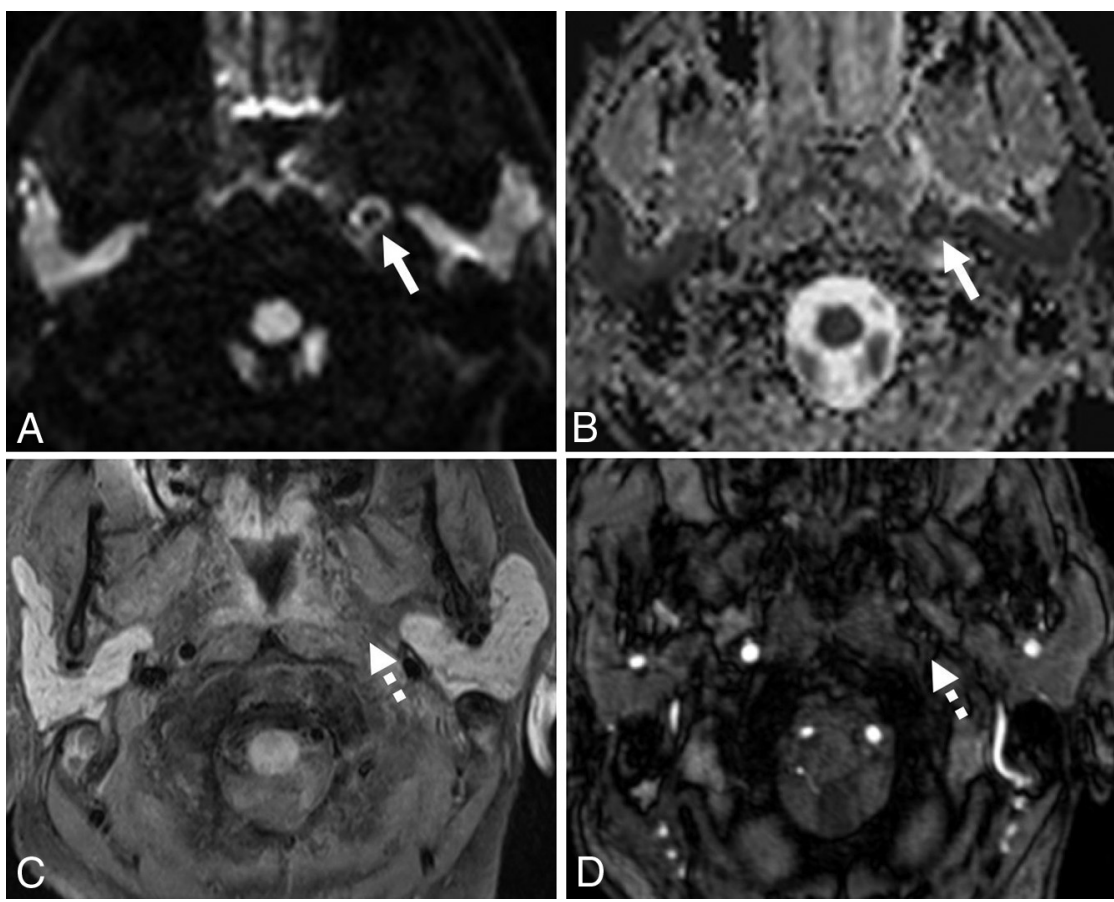

FIG 3. Hyperacute dissection with a wall hematoma appearing hyperintense on DWI (A, arrow), with apparent diffusion coefficient restriction ( $B$, arrow), not visible on FS TIWI (C, dotted arrow) or the axial source image of time-of-flight MR angiography ( $D$, dotted arrow). artery dissections, with the intensity of the MR imaging signal being directly proportional to the intensity of the primary magnetic field, the signal-tonoise ratio is higher on a sequence performed at $3 \mathrm{~T}$ than at $1.5 \mathrm{~T}$. It is likely that the wall hematomas of our series were more visible on $3 \mathrm{~T}$. Several studies have also shown interest in $3 \mathrm{~T}$ MR imaging using T1 Cube (GE Healthcare $)^{18}$ or black-blood ${ }^{19}$ sequences for detecting wall hematomas of dissections, but without comparing them with $1.5 \mathrm{~T}$.

In our study, DWI at $3 \mathrm{~T}$ revealed a parietal hematoma of ICA dissection very early because most of our patients with a dissection were examined by MR imaging within 24 hours after the onset of their symptoms. DWI was even better than FS T1WI in detecting wall hematoma in $15 \%$ of our patients, having undergone both sequences on their initial MR imaging performed, on average, 15 hours 20 minutes after the onset of symptoms. On the other subpetrous segments of the internal carotid arteries. Curiously, there are scant data in the literature regarding the accurate frequency of damage of the subpetrous segment in carotid dissections, even if it appears to be affected "in most cases."14 According to some authors, dissections usually originate $2 \mathrm{~cm}$ above the carotid bulb and extend over a variable length, often up to the carotid canal, covering the subpetrous segment. ${ }^{15}$

In our study, 1 dissection of 10 was not diagnosed by DWI, either due to the absence of hyperintensity despite a subpetrous topography included in the field of acquisition or because the hematoma was likely situated lower in the wall of the cervical ICA. The association between the tortuosity of cervical arteries and dissections is known. ${ }^{16}$ In 1 study, ${ }^{17}$ these loops were shown in $22 \%$ of dissections compared with $8 \%$ in healthy controls. In view of our results, these loops were often subpetrous, therefore included in the field of exploration, but some might be postbulbar and, therefore, not included in the field, and this feature may explain some of the false-negatives of the routine DWI brain sequences.

Among cervical artery dissections reported in the study of Naggara et al, ${ }^{1} 67$ ICA dissections were included in the fields of acquisition of brain MRIs and 21 carotid dissections were excluded, $76 \%$ of high dissections. This determination was made by analyzing axial FS T1WI performed on 1.5T magnets. Compared with our results, which indicate $90 \%$ of ICA dissections seen on subpetrous segments, it is possible that the findings of their sequence were faulty, particularly for the small wall hematomas or hematomas seen early, before 72 hours, and there were, in actuality, more carotid dissections included in the fields of acquisition of brain MRIs but not detected by their restrictive method. Although no studies have shown the superiority of 3T MR imaging compared with $1.5 \mathrm{~T}$ in the detection of cervical hand, the later the MRIs were performed, the more likely it was that DWI findings were faulty. The hyperintensity of the wall hematoma was only visible on FS T1WI in 2/26 (8\%) patients; these MRIs were then performed, on average, 6 days after the onset of symptoms (range, 5-7 days). The intracerebral hematoma signal intensity on DWI is the lowest during the early subacute phase of intracellular methemoglobin (between 4 and 7 days) before increasing again in the late subacute phase (after 7 days). ${ }^{11}$ This phenomenon could, therefore, explain the poorer sensitivity of DWI in detecting dissecting hematomas during this subacute phase.

The DWI and FS T1WI sequences, therefore, appear complementary in detecting ICA dissections: DWI could detect wall hematomas in the acute phase, even before the appearance of a FS T1WI hypersignal, while the latter could enable diagnosis of dissections beyond 72 hours and later than DWI. These considerations about the temporality are accurate if the wall hematoma occurs at the same time as the first symptoms of patients, which is not always the case.

Our study showed not only the very good sensitivity of DWI in the detection of ICA dissections but also an excellent specificity with no false-positives. Crescent- or ring-shaped hypersignals were likely to represent wall hematomas of dissections, not hemorrhagic plaques, because these are not supposed to appear with DWI hypersignal ${ }^{20}$ and are not usually located in the subpetrosal aspect of the distal ICA. However, it is important to learn to distinguish crescent- or ring-shaped dissections of hematomas from linear and nodular hyperintensities of pharyngeal lymphoid tissue and lymph nodes. Once this sign is learned, it enables detection of wall hematomas with excellent specificity and excellent interobserver agreement. In clinical routine, the radiologist will 
naturally be aided by other classic protocol sequences such as FLAIR and gradient recalled-echo $\mathrm{T}^{*}$ to more easily locate the hypersignal on DWI. However, in practice, in view of our results, if the hematoma clearly appears hyperintense on DWI at the acute phase of a neurologic deficit or neck pain, then the specificity of our sign can be obtained without FS T1WI and thus save acquisition time. It also does not appear necessary to re-examine the patient later to confirm the presence of a dissection if the hypersignal of the hematoma is visible in DWI on the initial MR imaging.

The primary limitation of our study is its retrospective nature, including 50 patients with dissections and 60 patients without dissections, a prevalence of $45 \%$ dissections in our total population, or an overrepresentation of dissections compared with the general stroke population. However, knowing this prevalence, if the sensitivity was increased, the observers would also have seen more hematomas than there were in reality, with a consequent decrease in specificity. This did not occur, as no false-positives were found.

Some patients with dissections had visible cerebral ischemic lesions on DWI. This could have been a bias in the analysis of the presence of a crescent- or ring-shaped hypersignal along the expected wall of the subpetrosal ICA, particularly if this hypersignal was on the same side as the ischemic lesions. This bias was removed by the same proportion of ischemic lesions in the dissection group as in the control group.

We chose to investigate ICA dissections; we do not know the sensitivity and specificity of DWI in the detection of vertebral artery dissections. One study ${ }^{12}$ observed DWI hyperintensity in connection with vertebral dissections in a small cohort of 4 patients on 1.5T MR imaging. A future study, including a greater number of patients and performed with 3T MR imaging will analyze the capacity of DWI in detecting wall hematomas of vertebral dissections. Finally, this following study may also compare the respective sensitivities and specificities of DWI and FS T1WI sequences in detecting cervical artery dissections as a function of the time of onset of symptoms.

\section{CONCLUSIONS}

In our study, a standard diffusion-weighted sequence routinely performed on the brain at the acute phase of a stroke or for a clinical suspicion of cervical artery dissection detected nearly $90 \%$ of cervical ICA dissections by showing a crescent- or ring-shaped hypersignal along the expected wall of the subpetrosal ICA. This sign, present on the first sequence generally acquired during MR imaging performed for a suspicion of acute ischemic stroke, makes it possible to confidently discuss the dissection diagnosis and initiate medical treatment adapted to very quickly reduce the risk of thromboembolic complications, and also to better predict the material necessary for a potential endovascular procedure.

Disclosures: Christophe Cognard-UNRELATED: Consultancy: Medtronic, Stryker, MicroVention, Cerenovus, MIVI. Zuzana Meluchova-UNRELATED: Employment: University Hospital Toulouse.

\section{REFERENCES}

1. Naggara O, Soares F, Touze E, et al. Is it possible to recognize cervical artery dissection on stroke brain MR imaging? A matched case- control study. AJNR Am J Neuroradiol 2011;32:869-73 CrossRef Medline

2. Schievink WI. Spontaneous dissection of the carotid and vertebral arteries. N Engl J Med 2001;344:898-906 CrossRef Medline

3. Biousse V, D'Anglejan-Chatillon J, Touboul PJ, et al. Time course of symptoms in extracranial carotid artery dissections: a series of $\mathbf{8 0}$ patients. Stroke 1995;26:235-39 CrossRef CrossRef Medline

4. Hoving JW, Marquering HA, Majoie C. Endovascular treatment in patients with carotid artery dissection and intracranial occlusion: a systematic review. Neuroradiology 2017;59:641-47 CrossRef Medline

5. Rotzinger DC, Mosimann PJ, Meuli RA, et al. Site and rate of occlusive disease in cervicocerebral arteries: a CT angiography study of 2209 patients with acute ischemic stroke. AJNR Am J Neuroradiol 2017;38:868-74 CrossRef Medline

6. Wei D, Mascitelli JR, Nistal DA, et al. The use and utility of aspiration thrombectomy in acute ischemic stroke: a systematic review and meta-analysis. AJNR Am J Neuroradiol 2017;38:1978-83 CrossRef Medline

7. Marnat G, Bühlmann M, Eker OF, et al. Multicentric experience in distal-to-proximal revascularization of tandem occlusion stroke related to internal carotid artery dissection. AJNR Am J Neuroradiol 2018;39:1093-99 CrossRef Medline

8. Bradley WG Jr. MR appearance of hemorrhage in the brain. Radiology 1993;189:15-26 CrossRef Medline

9. Bisseret D, Khalil A, Favrole P, et al. Spontaneous cervical artery dissection: role of a SE-T1-weighted fat-sat volume acquisition. Diagn Interv Imaging 2014;95:443-46 CrossRef Medline

10. Provenzale JM, Morgenlander JC, Gress D. Spontaneous vertebral dissection: clinical, conventional angiographic, CT, and MR findings. J Comput Assist Tomogr 1996;20:185-93 CrossRef Medline

11. Silvera S, Oppenheim C, Touzé E, et al. Spontaneous intracerebral hematoma on diffusion-weighted images: influence of T2-shinethrough and T2-blackout effects. AJNR Am J Neuroradiol 2005;26: 236-41 Medline

12. Choi KD, Jo JW, Park KP, et al. Diffusion-weighted imaging of intramural hematoma in vertebral artery dissection. J Neurol Sci 2007;253:81-84 CrossRef Medline

13. Haussen DC, Henninger N, Selim M. Diffusion-weighted imaging of intramural hematoma in internal carotid artery dissection. Acta Neurol Belg 2013;113:109-10 CrossRef Medline

14. Ben Hassen W, Machet A, Edjlali-Goujon M, et al. Imaging of cervical artery dissection. Diagn Interv Imaging 2014;95:1151-61 CrossRef Medline

15. Guillon B, Bousser MG. Epidemiology and pathophysiology of spontaneous cervical artery dissection. J Neuroradiol 2002;29:24149 Medline

16. Kim BJ, Yang E, Kim NY, et al. Vascular tortuosity may be associated with cervical artery dissection. Stroke 2016;47:2548-52 CrossRef Medline

17. Kim ST, Brinjikji W, Lehman VT, et al. Association between carotid artery tortuosity and carotid dissection: a case-control study. $J$ Neurosurg Sci 2018;62:413-17 CrossRef Medline

18. Edjlali M, Roca P, Rabrait C, et al. 3D fast spin-echo T1 black-blood imaging for the diagnosis of cervical artery dissection. AJNR Am J Neuroradiol 2013;34:E103-06 CrossRef Medline

19. Hunter MA, Santosh C, Teasdale E, et al. High-resolution double inversion recovery black-blood imaging of cervical artery dissection using 3T MR imaging. AJNR Am J Neuroradiol 2012;33:E13337 CrossRef Medline

20. Yao B, Yang L, Wang G, et al. Diffusion measurement of intraplaque hemorrhage and intramural hematoma using diffusion weighted MRI at 3T in cervical artery. Eur Radiol 2016;26:3737-43 CrossRef Medline 